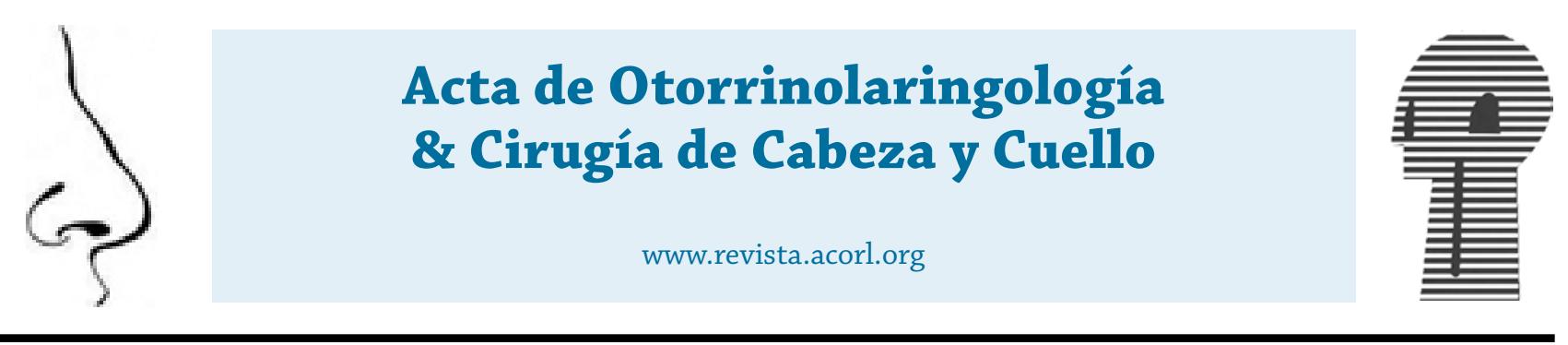

\title{
Caracterización de la población que consulta por epistaxis al servicio de otorrinolaringología del Hospital Universitario Mayor Méderi (HUM)
}

\section{Characterization of the population that consults by epistaxis to the Otolaryngology Service of the Hospital Universitario Mayor Méderi (HUM)}

\author{
Liliana Isabel Alfaro Arias*, Mary Lourdes Hernández López, ** Jóse Alejandro Daza Vergára*** \\ * Médica Otorrinolaringóloga, Departamento Otorrinolaringología, Hospital Universitario Mayor Méderi. \\ ** Médica Otorrinolaringóloga, Departamento Otorrinolaringología, Hospital Universitario Mayor Méderi. \\ *** Médico Epidemiólogo, Hospital Universitario Mayor Méderi.
}

Forma de citar: Alfaro-Arias LI, Hernández-López ML, Daza Vergára JA. Caracterización de la población que consulta por epistaxis al servicio de otorrinolaringología del Hospital Universitario Mayor Méderi (HUM). Acta otorrinolaringol. cir. cabeza cuello. 2020; 48(3):232-239 Doi:10.37076/acorl.v48i3.493

\section{INFORMACIÓN DEL ARTÍCULO}

Historia del artículo:

Recibido: 28 de abril de 2020

Evaluado: 24 de agosto de 2020

Aceptado: 28 de agosto de 2020

Palabras clave (DeCS):

epistaxis, demografía, terapeútica, hipertensión.

\section{RESUMEN}

Introducción: la epistaxis es uno de los principales motivos de consulta por urgencias en el servicio de otorrinolaringología (ORL). La mayoría de los episodios se originan en el septo nasal anterior y su tratamiento tiende a ser conservador. Objetivo: caracterizar la población que consulta por epistaxis al servicio de otorrinolaringología del Hospital Universitario Mayor Méderi (HUM) entre marzo de 2017 y febrero de 2018. Metodología: estudio observacional, descriptivo y de corte transversal, con una recolección de datos de forma retrospectiva de pacientes que ingresan a urgencias del HUM por epistaxis, registrados en la base de datos del servicio de otorrinolaringología. Se analizaron datos demográficos, localización del sangrado, tratamiento, comorbilidades, fármacos y cifras tensionales. Resultados: un total de 587 pacientes; el $57 \%$ son mujeres, con un promedio de 66 años $+/-16,15$ años. La comorbilidad más frecuente fue la hipertensión arterial $(60 \%)$ y el $26 \%$ de los 
pacientes presentaron cifras tensionales $>140 / 90 \mathrm{~mm} \mathrm{Hg}$. En antecedentes farmacológicos, el 64,9\% pertenecían al grupo de antihipertensivos y el 37,6 \% a los antiagregantes. La localización de los sangrados fue anterior ( $>$ área II de Cottle). El principal tratamiento fue el taponamiento nasal anterior con gasa (36,4\%), seguido por la cauterización con nitrato de plata $(36,1 \%)$. Conclusiones: en el HUM, los pacientes que consultan por epistaxis son adultos de edad media y avanzada, cuyo sangrado nasal tiene una localización anterior, que solamente requirieron tratamiento médico. Se requieren otros estudios para determinar la modalidad de tratamiento más efectiva según la gravedad de la epistaxis.

\section{ABSTRACT}

Key words (MeSH):

epistaxis, demography, therapeutics, hypertension.
Background: epistaxis is one of the main reasons for emergency consultations in the Otolaryngology department. Most episodes originate in the anterior nasal septum and their management tends to be conservative. Aim: characterize the population that consults by epistaxis to the Otolaryngology department of the Hospital Universitario Mayor Méderi (HUM) between March 2017 and February 2018. Methods: observational, descriptive, cross-sectional study, with retrospective data collection of patients admitted to the emergency department of the HUM by epistaxis, registered in the database of the Otolaryngology Department. Demographic data, location of bleeding, treatment, comorbidities, drugs, and blood pressure were analyzed. Results: a total of 587 patients; $57 \%$ women with an average of 66 years $+/-16.15$ years. The most frequent comorbidity was arterial hypertension (60\%) and $26 \%$ of the patients presented blood pressure levels $>140 / 90 \mathrm{~mm} \mathrm{Hg}$. In pharmacological history, $64.9 \%$ belonged to the group of antihypertensives and $37.6 \%$ to antiplatelet agents. The location of the bleeds was anterior ( $>$ Cottle area II). The main treatment was the anterior nasal gauze packing (36.4\%), followed by silver nitrate cauterization (36.1\%). Conclusions: in the HUM, patients who consult for epistaxis are middle-aged and elderly adults, with anterior localization of nasal bleeding, which only required medical management. Other studies are required to determine the most effective treatment modality according to the severity of epistaxis.

\section{Introducción}

La epistaxis es el sangrado que ocurre en la cavidad nasal y representa una de las causas más frecuentes de consulta en el servicio de urgencias, así como la causa más frecuente de ingreso al servicio de otorrinolaringología (1). Dos tercios de la población han presentado por lo menos un episodio de epistaxis durante su vida, de los cuales el $6 \%$ requieren atención médica (2). Además, cuenta con dos picos de presentación: el primero en pacientes jóvenes $<10$ años, (3) y el segundo en pacientes entre los 45-75 años (4).

En el Hospital Universitario Mayor Méderi (HUM), la epistaxis es uno de los motivos de consulta más frecuentes por otorrinolaringología, con un alto flujo de pacientes con múltiples comorbilidades y en polifarmacia. Hasta el momento, no se conocen con certeza las características demográficas y clínicas de esta población. El abordaje apropiado de los pacientes con epistaxis se logra conociendo de antemano las características demográficas de la población, la principal localización del sangrado, el tratamiento más efectivo, las comorbilidades y los medicamentos que puedan ocasionar un aumento del sangrado, y si el control de las cifras tensionales es un factor aditivo en el control de la epistaxis.
El objetivo del estudio es caracterizar la población que consulta por epistaxis al servicio de otorrinolaringología del HUM entre marzo de 2017 y febrero de 2018.

\section{Metodología}

Estudio observacional, descriptivo de corte transversal, en el que se realizó una recolección de datos de forma retrospectiva entre marzo de 2017 a febrero 2018 en el HUM. Se consideró tomar la totalidad de los registros clínicos de los pacientes ingresados durante un año al servicio de otorrinolaringología del HUM.

\section{Criterios de inclusión}

Se incluyeron pacientes mayores de edad atendidos en el servicio de otorrinolaringología con diagnóstico de epistaxis (código CIE 10: R040), que se encuentran en observación en el servicio de urgencias o en hospitalización del HUM entre marzo de 2017 y febrero de 2018; son pacientes del régimen contributivo o subsidiado, procedentes principalmente del centro del país. 


\section{Criterios de exclusión}

Se excluyeron pacientes con epistaxis secundaria a trauma nasal, tumores, pacientes embarazadas e historias clínicas incompletas, que no cuenten con los datos requeridos para el estudio.

\section{Método de recogida de datos}

Inicialmente, se seleccionan los sujetos que cumplieran con los criterios de inclusión de la base de datos de pacientes interconsultados al servicio de otorrinolaringología. Por protocolo de la institución, todos los pacientes firman un consentimiento informado al momento de ingresar al hospital, en el cual se incluye la autorización del uso de la información clínica con fines de investigación. En la toma de datos, se elabora un formato con las variables para cada paciente, en el que se encuentra la edad, el sexo, la localización del sangrado (por rinoscopia o endoscopia nasal), que se registra en anterior o posterior, unilateral o bilateral y el área de Cottle comprometida del septo nasal. Se incluyen las modalidades de tratamiento utilizadas, los trastornos médicos concurrentes, las cifras tensionales al momento del ingreso, si se realizó un International Normalized Ratio (INR), recuento de plaquetas y los medicamentos que usa el paciente a la fecha del episodio de sangrado nasal. Las modalidades de tratamiento identificadas incluyeron conservador ( $\sin$ tratamiento), presión nasal, taponamiento con gasa furacinada, taponamiento posterior con sonda de Foley, hemostático absorbible (Surgicel-Spongostan), cauterización con nitrato de plata, cirugía endoscópica o embolización arterial.

La información de cada paciente se tomó de las historias clínicas de la plataforma SERVINTE del HUM.

\section{Gestión informática de los datos}

Se cuenta con la base de datos del servicio de otorrinolaringología del HUM, en la se registran todos los pacientes interconsultados al servicio con el diagnóstico de epistaxis. Con base en la información allí consignada, se buscan las historias clínicas en el software SERVINTE, se toma la información requerida para el estudio y se registra en un formato, posterior a lo cual se trasladan los datos a una tabla de Microsoft Excel para su análisis.

\section{Análisis}

Los datos se procesaron de manera digital usando el programa de Microsoft Excel. Se digitalizaron los datos recolectados de los pacientes entre marzo de 2017 y febrero de 2018 con las variables ya enunciadas previamente, además, se realizó una digitación doble de los datos para el control de calidad. Una vez culminada la recolección de los datos, se pasó la base a la versión 24 del programa SPSS, con licencia de la Universidad del Rosario, con el cual se verificó la calidad de la información con los filtros necesarios; en caso de encontrar discrepancias en más del $20 \%$ de los datos, se realizó una redigitación. Para las estadísticas descriptivas y variables cuantitativas, se hizo inicialmente un análisis de normalidad con los las pruebas estadísticas Shapiro-Wilks o Kolmogórov-Smirnov, dependiendo del número de registros; para las variables normales se utilizó la media con su respectiva desviación estándar, para las variables no normales se utilizaron la mediana y el rangos intercuartílico, y para las variables cualitativas se utilizaron las frecuencias absolutas y relativas.

\section{Consideraciones éticas}

Según la Resolución No. 008430 de 1993, por la cual se establecen las normas científicas, técnicas y administrativas para la investigación en salud en Colombia, este estudio se cataloga como una investigación sin riesgo (5), ya que se utilizaron datos recolectados de las historias clínicas de los pacientes para la realización del estudio, sin necesidad de intervenir sobre los pacientes objeto de estudio y, de esta forma, sin posibilidad de ejercer daño sobre estos. En este estudio prevalece el criterio de respeto a la dignidad de los pacientes y la protección de sus derechos y su bienestar (6), mediante la protección a la privacidad del paciente sujeto de investigación (7).

\section{Resultados}

En el período comprendido entre marzo de 2017 y febrero de 2018, 3500 pacientes fueron remitidos a interconsulta al servicio de ORL. Se recolectó información de un total de 587 pacientes, que se valoraron por epistaxis en este año; de estos, el $57 \%$ eran mujeres con una edad promedio de 66 años (desviación estándar [DE]: 16,16) (Tabla 1). Esta información solo se tomó para el registro y el análisis del primer ingreso en los pacientes que consultaron más de una vez.

Tabla 1. Características generales de la población con
epistaxis
\begin{tabular}{|l|c|c|}
\hline Características población & $\mathrm{n}$ & $\%$ \\
\hline Total de registros evaluados & 587 & 100 \\
\hline Sexo & 335 & 57,1 \\
\hline Femenino & 252 & 42,9 \\
\hline Masculino & & \\
\hline Edad en años & 66 & \\
\hline Promedio & 18 & \\
\hline Mínimo & 96 & \\
\hline Máximo &
\end{tabular}

Del total de los pacientes, el 84,8 \% tenía alguna comorbilidad (Tabla 2); la más común se estaba relacionada con el grupo de enfermedades cardiovasculares, como hipertensión arterial (60\%), enfermedad coronaria, diabetes mellitus, insuficiencia cardíaca, etc. (Figura 1). 
Tabla 2. Presencia de comorbilidades en los pacientes con epistaxis

\begin{tabular}{|l|c|c|}
\hline \multicolumn{1}{|c|}{ Comorbilidades } & n & $\%$ \\
\hline Sí & 498 & 84,84 \\
\hline No & 89 & 15,16 \\
\hline Total de registros evaluados & 587 & 100 \\
\hline
\end{tabular}

En cuanto a los medicamentos que usaban los pacientes al momento de presentar el episodio de sangrado nasal, se encontró que el 64,9 \% pertenecían al grupo de los antihipertensivos (losartán, enalapril, amlodipino, furosemida, metoprolol) y el 37,6 \% a los antiagregantes (ASA, clopidogrel) (Figura 2).

Además, se encontró que en casi la totalidad de los pacientes la principal área de sangrado nasal fue la epistaxis anterior. Menos del $1 \%$ de los pacientes (3 pacientes) requirieron hospitalización por presentar sangrado posterior (Tabla 3).
Tabla 3. Clasificación de la epistaxis en la población del estudio

\begin{tabular}{|l|c|c|}
\hline \multicolumn{1}{|c|}{ Clasificación de la epistaxis } & $\mathbf{n}$ & $\%$ \\
\hline Anterior & 584 & 99,49 \\
\hline Posterior & 3 & 0,51 \\
\hline Total de registros evaluados & 587 & 100 \\
\hline
\end{tabular}

En cuanto a las áreas de Cottle del septo nasal, la epistaxis se presentó principalmente en el área II, que corresponde al área valvular (Figura 3).

En el $83 \%$ de los pacientes a quienes se les realizó una rinoscopia anterior, no se documentaron alteraciones anatómicas intranasales, y en los pacientes con hallazgos positivos, las deflexiones se documentaron en el $7 \%$ y las crestas basales en el 6,5\% (Figura 4)

De los procedimientos realizados para el manejo de la epistaxis, el taponamiento nasal anterior con gasa impregnada en nitrofurazona y la cauterización con nitrato de plata

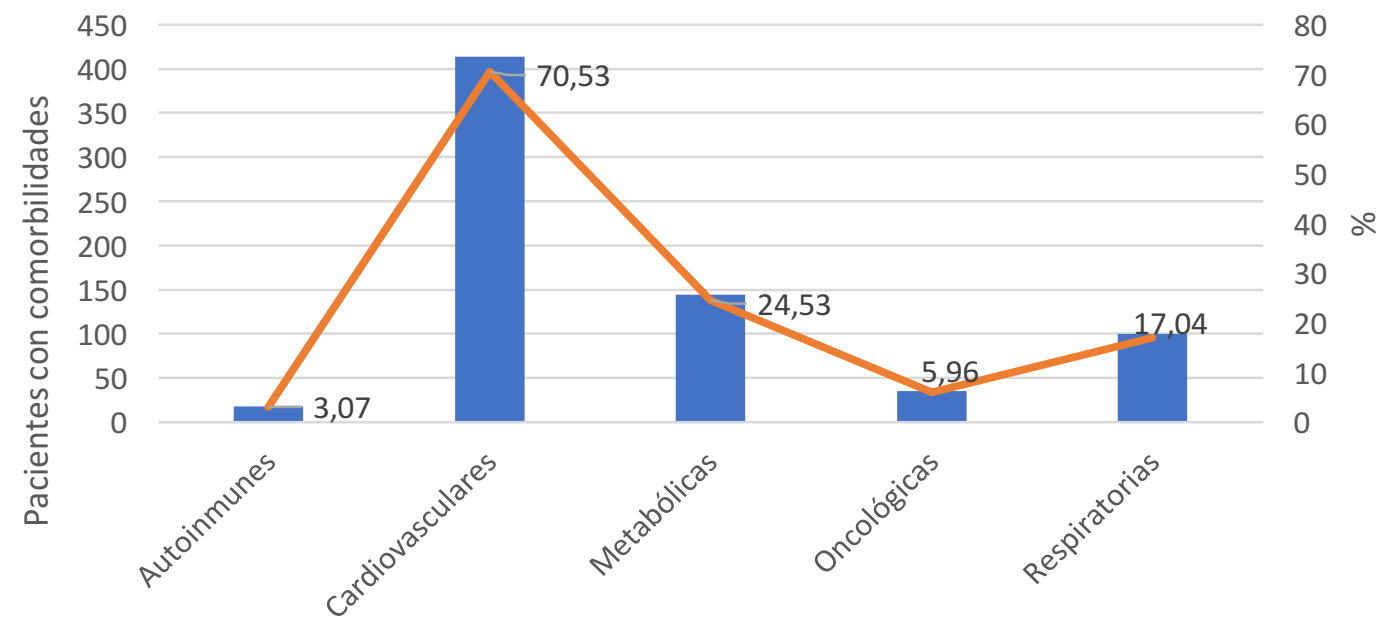

Figura 1. Comorbilidades de los pacientes con epistaxis.

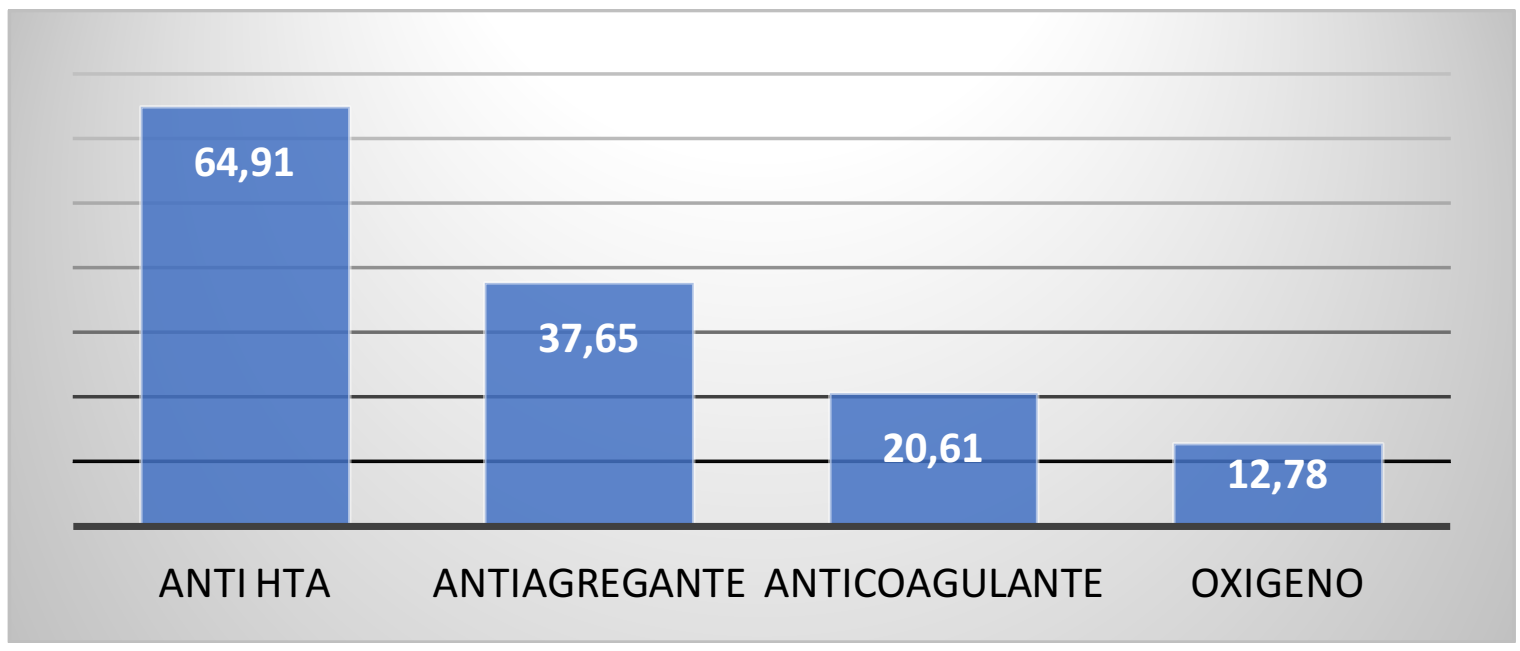

Figura 2. Medicamentos que consumen los pacientes con epistaxis. 


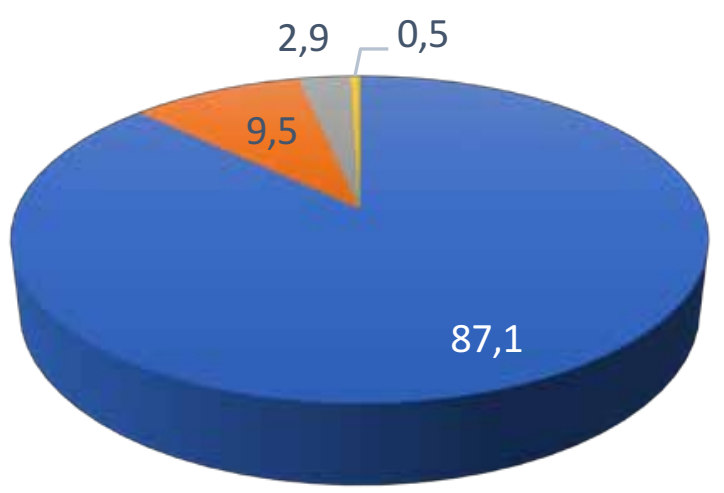

II $\square$ III $\square$ IV $\square$

Figura 3. Áreas del septo nasal donde se presentó la epistaxis (según la clasificación de Cottle).

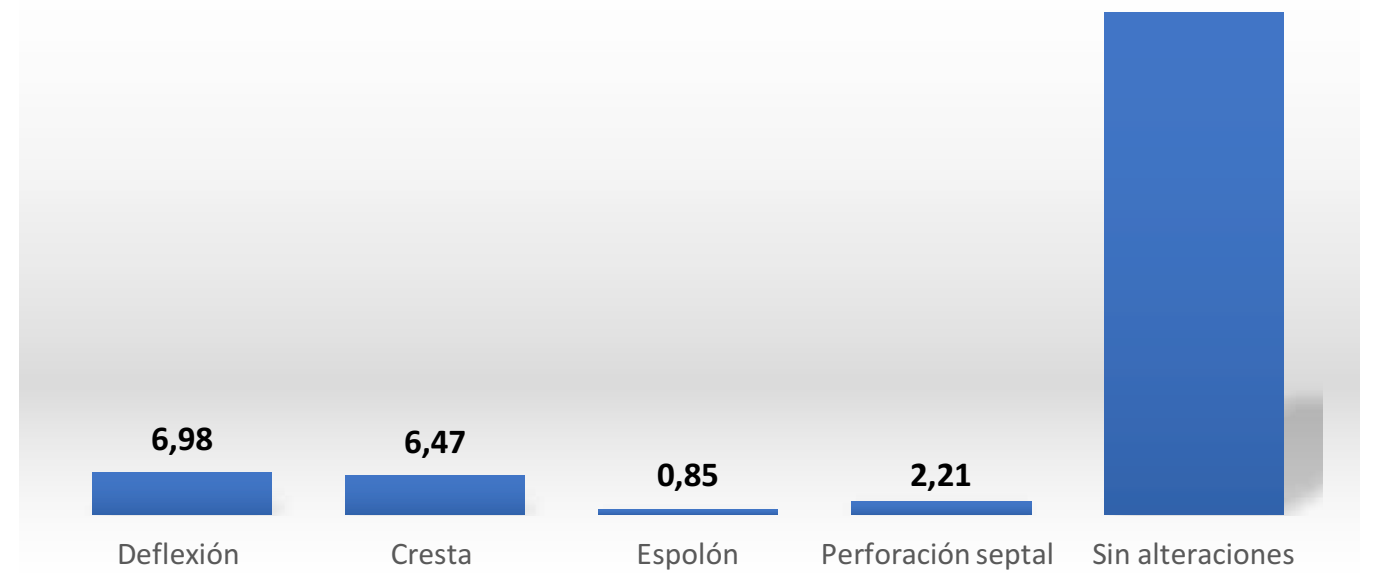

Figura 4. Alteraciones anatómicas intranasales en los pacientes con epistaxis.

fueron los principales tratamientos usados en el grupo de pacientes analizados (Figura 5).

Las cifras de presión arterial encontradas en los registros de los pacientes al momento de presentar el episodio de epistaxis fueron, en promedio, de $140 \mathrm{~mm} \mathrm{Hg}$ (DE: 26,5 $\mathrm{mm} \mathrm{Hg}$ ) para la presión arterial sistólica y $80 \mathrm{~mm} \mathrm{Hg}$ (DE: 14,6 mm Hg) para la presión arterial diastólica (Tabla 4). En esta medida, se encontró que el $26 \%$ presentaron cifras tensionales iguales o mayores a 140/90 mm Hg.

A 39 pacientes se les documentó la realización del INR al momento de la consulta por antecedentes de anticoagulación con warfarina. Se encontró que, de estos pacientes, el $71,7 \%$ se encontraban anticoagulados o sobreanticoagulados (Tabla 5).

A 43 pacientes se les realizó un cuadro hemático por antecedentes de comorbilidad oncológica o autoinmune, y se documentó que el $83 \%$ de estos pacientes presentaban trombocitopenia grave (Tabla 6).
Tabla 4. Presión arterial sistólica y diastólica en los pacientes con epistaxis

\begin{tabular}{|l|c|}
\hline Promedio & 140 \\
\hline Mínimo & 85 \\
\hline Máximo & 230 \\
\hline Presión arterial diastólica & $\mathrm{mm} \mathrm{Hg}$ \\
\hline Promedio & 80 \\
\hline Mínimo & 50 \\
\hline Máximo & 150 \\
\hline
\end{tabular}

\section{Discusión}

La epistaxis representa una de las causas más frecuentes de consulta en el servicio de urgencias, siendo aproximadamente entre el 9,5-24,5\% de los ingresos totales $(1,8)$ y de los ingresos al servicio de otorrinolaringología. En Estados Uni- 


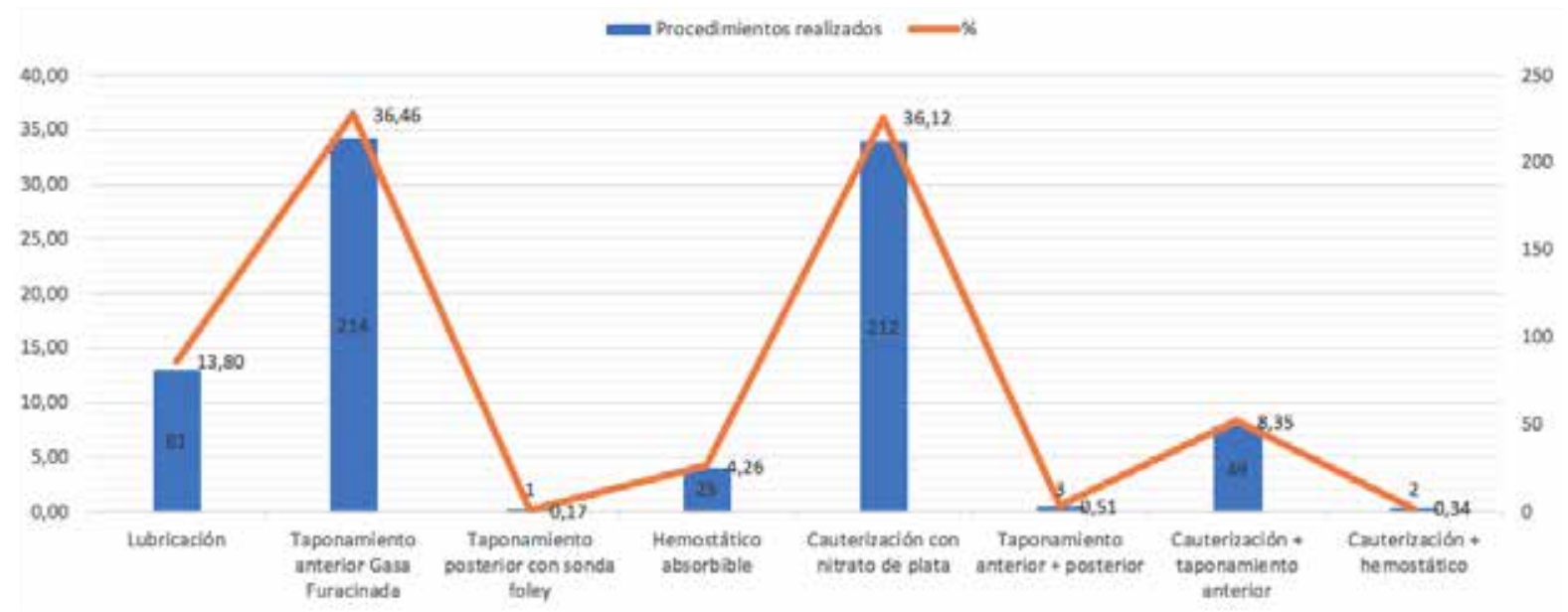

Figura 5. Tratamiento realizado a los pacientes con epistaxis.

Tabla 5. Valor del INR en los pacientes con epistaxis

\begin{tabular}{|l|c|c|}
\hline \multicolumn{1}{|c|}{ INR } & $\mathbf{n}$ & $\%$ \\
\hline 1,2-1,9: no anticoagulado & 11 & 28,21 \\
\hline 2-3: anticoagulado & 13 & 33,33 \\
\hline >3: sobreanticoagulado & 15 & 38,46 \\
\hline
\end{tabular}

Tabla 6. Valor del recuento de plaquetas en los pacientes con epistaxis

\begin{tabular}{|l|c|c|}
\hline \multicolumn{1}{|c|}{ Plaquetas } & $\mathrm{n}$ & $\%$ \\
\hline$>\mathbf{1 5 0}$ & 1 & 2,33 \\
\hline $\mathbf{5 0 - 1 5 0}$ & 6 & 13,95 \\
\hline$<50$ & 36 & 83,72 \\
\hline
\end{tabular}

dos constituye alrededor de 1 de cada 200 visitas a urgencias (9). Aproximadamente el $60 \%$ de la población general presenta al menos un episodio de epistaxis a lo largo de la vida, y el $6 \%$ de estos pacientes requieren atención médica por presentar hemorragia significativa no controlada (10).

En un período de un año, nuestro servicio de otorrinolaringología valoró aproximadamente a 3500 pacientes de los servicios de urgencias y hospitalización del HUM, de los cuales 587 casos fueron diagnosticados y valorados en nuestro servicio como epistaxis (17\%), cifras ligeramente mayores a la información encontrada en la literatura $(8,9)$. En la mayoría de los casos, la epistaxis puede tratarse de forma ambulatoria con medidas de control en una sala de procedimientos de otorrinolaringología, sin requerir hospitalización ni la realización de procedimientos quirúrgicos. Como se evidencia en este estudio, solo 3 pacientes $(0,51$ $\%$ requirieron taponamiento posterior y hospitalización a corto plazo.

Los adultos mayores de 60 años son la población que más consulta $(73 \%)$ al servicio de otorrinolaringología, datos también obtenidos en otros estudios $(9,11)$. No se encontró una diferencia significativa en el sexo, el 57\% de la población pertenece al sexo femenino, de manera similar al estudio publicado por Corr y colaboradores (12); estos datos no son constantes en la literatura, ya que la mayoría de las publicaciones muestran un predominio del sexo masculino $(8,13)$, pero a partir de los 50 años la incidencia tiende a ser igual tanto en hombres como mujeres, como lo muestra nuestro estudio y otras publicaciones (8). La mayoría de los sangrados nasales se localizan en la zona anterior de la mucosa septal, principalmente en el área II de Cottle. Ninguno de los pacientes requirió de un tratamiento quirúrgico dentro del período evaluado, lo que se relaciona con lo mencionado anteriormente; la mayoría de los pacientes pueden ser tratados de forma ambulatoria y con recomendaciones generales como compresión del área correspondiente al plexo de Kiesselbach y lubricación, tal como se concluyó en el estudio de Kubba y colaboradores (14).

Escoger el tratamiento ideal para el control de la epistaxis, entre el taponamiento anterior con materiales no absorbibles y la cauterización química, depende generalmente de la visualización o no del sitio del sangrado y la cuantía de este. El taponamiento anterior con gasa impregnada en antibiótico y la cauterización con nitrato de plata fueron las modalidades más utilizadas para el control del sangrado, lo cual es efectivo y representa el $72 \%$ del tratamiento inicial, similar a los resultados mostrados en otros estudios como el de Newton y colaboradores (13) y Corr y colaboradores (12).

Se han descrito múltiples factores de riesgo para la epistaxis, pero la gran mayoría son controvertidos; entre estos, los medicamentos han generado ciertas discusiones científicas. Nuestro hospital atiende a pacientes de alta complejidad y usuarios frecuentes de múltiples medicamentos, quienes tienen una alta probabilidad de presentar sangrados, lo cual altera la hemostasia. Es así como el $37 \%$ de los pacientes recibieron antiagregantes, siendo los más frecuentes la aspirina y el clopidogrel, y $20 \%$ usan anticoagulantes como la warfarina y la enoxaparina.

La relación entre epistaxis e hipertensión también es controvertida. Algunos estudios muestran una relación positiva (15, 16) y otros ninguna relación (17), por lo que en la actualidad no existe evidencia suficiente para afirmar que la hipertensión arterial sea un factor de riesgo para la epistaxis. Un metaaná- 
lisis publicado recientemente, que evaluó la asociación entre hipertensión y epistaxis (18), concluye que la hipertensión está significativamente asociada con el riesgo de epistaxis; sin embargo, existe una heterogeneidad significativa. Nuestro estudio muestra que el $60 \%$ de los pacientes presentaron diagnóstico de hipertensión arterial; un hallazgo importante es que el $26 \%$ presentaron cifras tensionales $>140 / 90 \mathrm{~mm} \mathrm{Hg}$ al momento de la valoración médica por el sangrado nasal, lo cual podría ser un factor de riesgo para el primer episodio y la recurrencia debido a que induce cambios ateroescleróticos y una menor elasticidad en los vasos sanguíneos.

A los pacientes anticoagulados con warfarina se les realizó un control con INR en el momento del episodio de la epistaxis, que mostró que el $71 \%$ se encontraban con niveles por encima de 2 , es decir, niveles de anticoagulación y sobreanticoagulación. Lo anterior podría estar relacionado con el riesgo de recurrencia de la epistaxis, gravedad de la epistaxis o ser un efecto aditivo de otros posibles factores de riesgo, como la hipertensión y el uso de antiagregantes (15).

\section{Conclusiones}

El patrón de los pacientes admitidos al servicio de otorrinolaringología del HUM que presentaron epistaxis es, en su mayoría, adultos de 60 y más años, con una ligera tendencia para el sexo femenino.

La localización del sangrado en esta población es anterior, principalmente en el área II de Cottle. Entre las modalidades de tratamiento más utilizadas se encontraron el taponamiento nasal anterior con gasa impregnada en nitrofurazona y la cauterización con nitrato de plata.

De las comorbilidades asociadas se encontró, principalmente, en el grupo cardiovascular, la hipertensión arterial, la diabetes mellitus y la insuficiencia cardíaca. Además, se encontró un porcentaje significativo de pacientes con cifras tensionales iguales o mayores a 140/90 $\mathrm{mm} \mathrm{Hg}$ durante el episodio de sangrado nasal.

El grupo de medicamentos más usado por los pacientes que presentaron epistaxis fue el de los antihipertensivos, debido a los factores de riesgo y las comorbilidades asociadas, $\mathrm{y}$ los antiagregantes plaquetarios.

Se requieren otro tipo de estudios para determinar la relación de estos hallazgos y definir los factores de riesgo para el primer episodio y recurrencia del sangrado, así como la modalidad de tratamiento más eficaz según la localización y la gravedad de la epistaxis.

\section{REFERENCIAS}

1. Bequignon E, Vérillaud $\mathrm{B}$, Robard L, Michel J, Prulière Escabasse V, Crampette L, et al. Guidelines of the French Society of Otorhinolaryngology (SFORL). First-line treatment of epistaxis in adults. Eur Ann Otorhinolaryngol Head Neck Dis. 2017;134(3):185-9. doi:10.1016/j.anorl.2016.09.008

2. Al-bar MH. Epistaxis and catastrophic nasal bleeding. Oper Tech Otolaryngol - Head Neck Surg. 2014;25(2):194-200. doi: 10.1016/j.otot.2014.02.010
3. Davies K, Batra K, Mehanna R, Keogh I. Pediatric epistaxis: epidemiology, management \& impact on quality of life. Int J Pediatr Otorhinolaryngol. 2014;78(8):1294-97. doi:10.1016/j. ijporl.2014.05.013

4. Kasperek ZA, Pollock GF. Epistaxis: an overview. Emerg Med Clin North Am. 2013;31(2):443-454. doi:10.1016/j. emc.2013.01.008

5. Kindler RM, Holzmann D, Landis BN, Ditzen B, Soyka MB. The high rate of long-term recurrences and sequelae after epistaxis treatment. Auris Nasus Larynx. 2016;43(4):412-7. doi:10.1016/j.anl.2015.09.011

6. Verillaud B, Robard L, Michel J, Pruliere Escabasse V, Béquignon E, et al. Guidelines of the French Society of Otorhinolaryngology (SFORL). Second-line treatment of epistaxis in adults. Eur Ann Otorhinolaryngol Head Neck Dis. 2017;134(3):191-193. doi:10.1016/j.anorl.2016.09.009

7. Lin G, Bleier B. Surgical Management of Severe Epistaxis. Otolaryngol Clin North Am. 2016;49(3):627-37. doi:10.1016/j. otc. 2016.01 .003

8. Monjas-Cánovas I, Hernández-García I, Mauri-Barberá J, SanzRomero B, Gras-Albert JR. Epidemiología de las epistaxis ingresadas en un hospital de tercer nivel. Acta Otorrinolaringol Esp. 2010;61(1):41-7.

9. Pallin DJ, Chng YM, McKay MP, Emond JA, Pelletier AJ, Camargo CA Jr. Epidemiology of epistaxis in US emergency departments, 1992 to 2001. Ann Emerg Med. 2005;46(1):7781. doi:10.1016/j.annemergmed.2004.12.014

10. Viehweg TL, Roberson JB, Hudson JW. Epistaxis: diagnosis and treatment. J Oral Maxillofac Surg. 2006;64(3):511-518. doi:10.1016/j.joms.2005.11.031

11. Ando Y, Iimura J, Arai S, et al. Risk factors for recurrent epistaxis: importance of initial treatment. Auris Nasus Larynx. 2014;41(1):41-45. doi:10.1016/j.anl.2013.05.004

12. Corr MJ, Tikka T, Douglas CM, Marshall J. One-year all-cause mortality for 338 patients admitted with epistaxis in a large tertiary ENT centre. J Laryngol Otol. 2019;133(6):487-493. doi:10.1017/S0022215119000860

13. Newton E, Lasso A, Petrcich W, Kilty SJ. An outcomes analysis of anterior epistaxis management in the emergency department. J Otolaryngol Head Neck Surg. 2016;45:24. doi:10.1186/ s40463-016-0138-2

14. Kubba H, MacAndie C, Botma M, et al. A prospective, singleblind, randomized controlled trial of antiseptic cream for recurrent epistaxis in childhood. Clin Otolaryngol Allied Sci. 2001;26(6):465-468. doi:10.1046/j.1365-2273.2001.00502.x

15. Abrich V, Brozek A, Boyle TR, Chyou PH, Yale SH. Risk factors for recurrent spontaneous epistaxis. Mayo Clin Proc. 2014;89(12):1636-1643. doi:10.1016/j.mayocp.2014.09.009

16. Terakura M, Fujisaki R, Suda T, Sagawa T, Sakamoto T. Relationship between blood pressure and persistent epistaxis at the emergency department: a retrospective study. J Am Soc Hypertens. 2012;6(4):291-295. doi:10.1016/j.jash.2012.05.001

17. Lubianca Neto JF, Fuchs FD, Facco SR, et al. Is epistaxis evidence of end-organ damage in patients with hypertension?. Laryngoscope. 1999;109(7 Pt 1):1111-15. doi:10.1097/00005537-199907000-00019

18. Min HJ, Kang H, Choi GJ, Kim KS. Association between Hypertension and Epistaxis: Systematic Review and Metaanalysis. Otolaryngol Head Neck Surg. 2017;157(6):921-927. doi:10.1177/0194599817721445 